\title{
Incentive contrast produced by deprivation shifts
}

\author{
ROBERT EISENBERGER \\ University of Delaware, Newark, Delaware 19711 \\ and
}

MICHAEL MIRSKY and MICHAEL FRANK

State University of New York at Albany, Albany, New York 12222

\begin{abstract}
Deprivation shifts, using conditions analogous to those which produce strong incentive contrast when reward quantity is changed (extended preshift training, short interval from the last preshift trial to the first postshift trial, large preshift differences in deprivation) resulted in contrast of runway speed and choice behavior. In the first experiment, a downshift of hunger during rats' runway training produced a slow lessening of speed below that of a group trained continually at low hunger. In the second experiment, rats were trained to traverse a runway to one food-containing goalbox when very hungry and to another, distinctively different food-containing goalbox when not very hungry. The rats were next given a series of choice trials between the two goalboxes. There was a brief preference for the high-hunger goalbox, followed by a preference for the low-hunger goalbox. The results of the second experiment suggest that deprivation affects the strength of conditioning of a cue-reinforcer expectancy, while the slow development of contrast in the first experiment indicates that deprivation also affects the development of either habit strength or a response-reinforcer expectancy.
\end{abstract}

Rats switched from high to low hunger following a small number of rewarded runway trials run faster on initial rewarded postshift trials than do animals trained all along in the state of less hunger (Brush, Goodrich, Teghtsoonian, \& Eisman, 1963; Butter \& Campbell, 1960; Capaldi, 1971; Capaldi \& Hovancik, 1973; Deese \& Carpenter, 1951; Eisenberger, Myers, \& Kaplan, 1973; Timberlake, 1967; but see Mollenauer, 1971). Associative and incentive-motivational interpretations of this result have been offered. One associative explanation assumes that the level of deprivation at which a stimulus-response sequence is followed by consumatory stimulation affects the asymptotic value of the stimulus' tendency to evoke the response (Capaldi, 1972; Eisenberger et al., 1973; Hovancik, 1978). Incentive-motivational interpretations hold that the level of deprivation at which consumatory stimulation occurs affects the strength of conditioning of reward expectancy to the exteroceptive and proprioceptive stimuli that precede the reward (Black, 1965; Bolles, 1958, 1975; Capaldi, Smith, \& White, 1978; Kurtz \& Jarka, 1968; Mollenauer, 1971; Moltz \& Maddi, 1956).

Negative incentive contrast refers to the finding that levels of performance are lower when reward

Reprint requests should be sent to Robert Eisenberger, Department of Psychology, University of Delaware, Newark, Delaware 19711. magnitude is decreased than when the lesser reward magnitude is employed all along. As with the downshift of deprivation level, the initial performance of animals receiving a reduction in reward during runway training is better than that of a group consistently receiving small reward. However, speed soon falls to a level less than that of the constant-smallreward group. Incentive motivational interpretations attribute this negative incentive-contrast effect to the occurrence of a reinforcing event that is of lesser magnitude than anticipated (Black, 1965; Bower, 1961). Failures to find contrast following downshifts of deprivation fail to support a simple analogy between shifts of reward quantity and shifts of the level of deprivation during instrumental performance.

Mollenauer (1971) pointed out that most deprivationshift experiments included relatively few preshift trials, although the magnitude of contrast produced by the downshift of reward quantity increases with the number of preshift trials (Vogel, Mikulka, \& Spear, 1966). Mollenauer gave rats a large number of preshift runway trials $(\mathrm{N}=75)$, with half the rats trained prior to 1 -h daily feeding sessions and the remaining rats trained following feeding. All animals then received an additional 20 rewarded trials, with half of each group shifted to the alternate level of hunger. Mollenauer claimed to have obtained contrast because the running speed of the downshifted group was slower than its preshift speed and slower 
than the test speed of the upshifted group, whereas performance prior to the shifts was not significantly affected by current hunger. However, the most appropriate comparison is between the downshifted group and the group run all along at low hunger, and this difference was not statistically significant (absence of a reliable interaction between postshift deprivation level and presence vs. absence of a shift). Mollenauer's failure to find reliable contrast may stem from not employing a strong enough hunger manipulation, as indicated by the absence of a statistically significant difference between groups in terminal preshift speed. Analogously, the magnitude of contrast produced by shifts of reward quantity has been found to be an increasing function of the preshift difference across groups in reward quantity (Di Lollo \& Beez, 1966).

Considering other experiments which employed a large number of preshift trials, Zaretsky (1966) failed to find contrast following deprivation downshift. However, Zaretsky's analysis was not the most sensitive possible, since it took into account only speed data obtained from the middle section of the runway. Capaldi (1973) noted that Zaretsky used small rewards (either a $45-\mathrm{mg}$ pellet or a $260-\mathrm{mg}$ pellet), while Mollenauer used a large reward ( $1.5 \mathrm{~g}$ of wet mash). Capaldi found that the use of small reward resulted in the retention of speeds that were faster following a shift from $75 \%$ body weight to $90 \%$ body weight than with continual maintenance at $90 \%$ body weight, whereas the same treatments with large reward resulted in the gradual development of contrast. Capaldi concluded that two conditions are necessary to obtain contrast produced by deprivation downshift: a large amount of preshift training and a large reward quantity.

Although the negative contrast reported by Capaldi is somewhat similar to that produced by reductions of reward quantity, the finding that deprivation downshift produced contrast only when large reward was used is not easily explained by the incentive motivational interpretation, since even small reward under high deprivation is very motivating and therefore should produce the strong conditioning of reward expectancy. Capaldi's establishment of differences in body weight for the hunger manipulation necessitated an 18-day interval from the last preshift trial to first postshift trial in order to equate hunger across groups. Since the magnitude of contrast is lessened when the interval from the last preshift trial to the first postshift trial is increased (Gleitman \& Steinman, 1964), the interval in Capaldi's experiment may have eliminated the development of some degree of contrast for small-reward animals. In the present experiments, all animals were maintained on the same deprivation cycle and the point on the cycle at which they were run was varied. Therefore, a short interval from the last preshift trial to the first postshift trial could be accomplished. The present research tested for contrast produced by deprivation shifts with small rewards, using conditions analogous to those which produce strong contrast when reward quantity is changed (extended preshift training, short training-test interval, large preshift difference in deprivation).

\section{EXPERIMENT 1}

\section{Method}

Subjects and Apparatus. Forty male Sprague-Dawley rats, approximately 100 days old, served as subjects. The animals were housed individually with constant 24 -h illumination. The apparatus consisted of a plywood straight alley $150.3 \times 12 \times 21.6 \mathrm{~cm}$. Opaque guillotine doors separated an 18.5-cm-long startbox from a $96-\mathrm{cm}$ runway and the runway from a $35.8-\mathrm{cm}$-long goalbox. The floors and walls of the startbox and runway were painted gray. The floors and walls of the goalbox were painted white. Raising of the startbox door closed a microswitch, which in turn activated a Hunter timer. A silent photoelectric relay stopped the timer when the animal broke a photocell beam that crossed the entrance to the goalbox.

Procedure. Throughout the experiment, all animals were maintained on a feeding cycle consisting of feeding periods of $22 \mathrm{~h}$ alternated with deprivation periods of $26 \mathrm{~h}$. At the start of each feeding period, a sufficient number of food pellets were placed on the floor of each cage such that some pellets always remained, and were removed, at the end of the feeding period. The subjects were gentled for $5 \mathrm{~min}$ on each of the first 2 days of pretraining. On the next 4 days, twice a day the animals were allowed to explore a two-compartment box. When the rat moved from the first compartment into the second, a guillotine door was lowered and the animal was kept in the second compartment for $3 \mathrm{~min}$. The second compartment contained a single $37-\mathrm{mg}$ Noyes pellet in a food cup. As a consequence of the feeding cycle, each animal received half of these preliminary trials under high deprivation and half received them under low deprivation. The subjects were next assigned to two groups, a deprivation-shift group, which was initially trained at $26 \mathrm{~h}$ deprivation and later at $2 \mathrm{~h}$ deprivation, and a constantlow group, which was trained continually at $2 \mathrm{~h}$ deprivation. For both groups, the intersession interval was $48 \mathrm{~h}$. All animals received one food-rewarded runway trial in the first experimental session, three trials in the second, and four trials in each of the next 27 sessions. The deprivation-shift group received 72 highdeprivation trials followed by 40 low-deprivation trials, while the constant-low group received all 112 trials under low deprivation. Since, for each animal, identical points on the feeding cycle were $48 \mathrm{~h}$ apart, the interval experienced by the deprivation-shift group from the last high-deprivation trial to the first low-deprivation trial could not be the usual $48 \mathrm{~h}$. In order to counterbalance for the duration of this interval in such a way that all shifted animals received their first postshift trial at the appropriate hunger level ( $2 \mathrm{~h}$ following the termination of the feeding period) and that the average interval of the deprivation-shift group from the last preshift trial to the first postshift trial was the same as that of the constant-low group ( $48 \mathrm{~h}$ ), half the deprivation-shift animals experienced a 24-h interval and the remainder, a 72-h interval. All subsequent postshift sessions were separated by the usual 48-h interval.

On each trial of a session, the rat was first placed in the startbox. Thirty seconds later, the startbox door was opened. As the rat traversed the alley, the startbox door and goalbox door were lowered to prevent retracing. After the rat entered the goalbox, the experimenter checked at $30-\mathrm{sec}$ interval up to $5 \mathrm{~min}$ to see if all of the four $37-\mathrm{mg}$ Noyes pellets in the food cup had been eaten. 
The animal was removed at the end of the $30-\mathrm{sec}$ interval in which it ate or, if it did not eat, at the end of $5 \mathrm{~min}$. The number of failures to completely consume the reward was negligible, and by the last trial of the fourth session every animal on every trial was completely consuming the reward within $30 \mathrm{sec}$. If another trial using the subject was scheduled, the animal was immediately replaced in the startbox and the preceding procedure was repeated. Otherwise, the animal was returned to its home cage.

\section{Results}

All statistical comparisons in this and the following experiment are two-tailed. Speed scores averaged over blocks of eight trials were employed for the statistical analyses in the first experiment. As illustrated in Figure 1, the difference of speed between the deprivation-shift group and the constant-low group increased over preshift trials. A $2 \times 9$ (group $\times$ trial block) repeated measures analysis of variance revealed significant effects for groups $[F(1,38)=7.26$, $\mathrm{p}<.05]$, trials $[\mathrm{F}(8,304)=93.1, \mathrm{p}<.001]$, and the groups $\times$ trials interaction $[F(8,304)=2.98, p<.005]$.

On the first block of trials following the deprivation shift, the two groups ran at about the same speed. The deprivation-shift group then gradually began to run more slowly than the constant-low group. These results are indicated by a significant group $x$ trials interaction $[F(4,152)=2.65, p<.05]$. Planned comparisons revealed that a significant difference between means was not present on the first block of postshift trials $[\mathrm{t}(38)=.25]$, but did occur on the last block of postshift trials $[\mathrm{t}(38)=2.05, \mathrm{p}<.05]$.

In accordance with the incentive-motivational interpretation, the use of conditions analogous to those which produce negative contrast when reward quantity is shifted (extended preshift training, large preshift differences in deprivation, and a short inter-

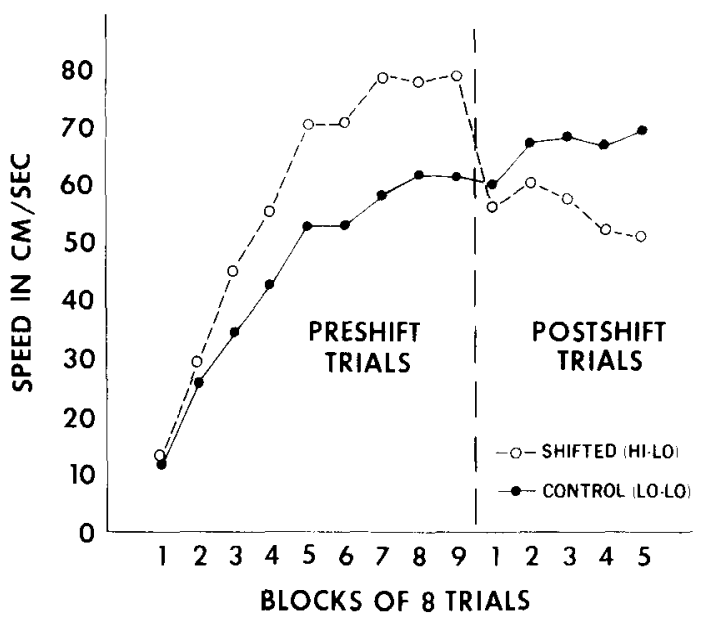

Figure 1. Mean running speed in blocks of eight trials throughout training for the group shifted from high to low hunger (deprivation-shift group) and the group run continually at low hunger (constant-low group). val from the last preshift trial to the first postshift trial) resulted in the lessening of the speed of the deprivation-shift group to a level below that of the constant-low group. However, unlike the contrast usually produced by shifts of reward quantity, the effect was slow to develop.

\section{EXPERIMENT 2}

Since the occurrence of contrast in the first experiment is not explained by an associative interpretation, and the gradual development of the effect is not easily explained by the incentive motivational account, it is possible that the level of deprivation at which reward is received affects both incentive motivation and habit strength. According to this view, a deprivation downshift following a small number of preshift trials would produce some immediate lessening of performance because of generalization decrements of habit strength and reward expectancy, but habit strength and reward expectancy would still be greater than they would be among subjects receiving low-deprivation trials all along. The greater initial postshift performance of the down-shifted group would gradually diminish as a result of adjustments of habit strength and incentive motivation to levels appropriate to the new degree of deprivation. Increasing the amount of preshift training would strengthen the conditioning of reward expectancy (Amsel, 1958; Peterson \& McHose, 1980; Spence, 1956) and, therefore, produce greater disconfirmation when the deprivation level was reduced. With many preshift trials and a short interval from the last preshift trial to the first postshift trial, negative contrast would develop following a deprivation shift, but only slowly, since generalized habit strength would operate in the opposite direction and gradually adjust to the new appropriate level.

The preceding interpretation assumes that habit strength slowly adjusts to the prevailing deprivation level at which a response is rewarded and may decrease if deprivation level is lowered. Another possibility is that the change of deprivation level affects only motivation. Bolles' (1972) expectancy interpretation of learning assumes that instrumental conditioning involves the acquisition of a cue-reinforcer expectancy and a response-reinforcer expectancy. Increasing the level of hunger at which a response is rewarded with food might result in a greater reward value of food and stronger cue-reinforcer and responsereinforcer expectancies. The contrast effect in the first experiment might have developed slowly because the response-reinforcer expectancy declined slowly and thereby operated in the direction opposite to the disruptive effects produced by the decrease in the value of the food reward. This argument supposes 
that the response-reinforcer expectancy is affected by deprivation level but not by the quantity of reward, the same assumption previously made for habit strength.

One way to separate the possible effects of deprivation upon a cue-reinforcer expectancy from habit strength or response-reinforcer expectancy is to retain the original conditioned stimuli (secondary rewards and/or cues) when deprivation level is shifted but impose a new instrumental requirement (cf. Hovancik, 1978; Peterson \& McHose, 1980). The performance of the new instrumental behavior would not be affected by the acquisition of the previous instrumental behavior. If deprivation level influenced the strength of conditioning of a cue-reinforcer expectancy, the presentation of the conditioned stimuli should result in an initial postshift performance that was greater in the downshifted group than in the constant-low-deprivation group, followed by contrast as the stronger cue-reinforcer expectancy by the downshifted group went unfulfilled.

The present experiment employed a runway for training and a U-maze for test in order to access the effects of deprivation upon the reward value of goalbox stimuli independent of habit strength or responsereinforcer expectancy. Eisenberger, Park, and Frank (1975) found that shifts of reward quantity produced contrast of choice behavior, and Spear and Spitzner (1966) found that the pairing of a shift of reward quantity with a new instrumental requirement produced contrast. In Experiment 2, rats were alternately given food-rewarded runway trials to one goalbox at high hunger and food-rewarded trials to a distinctively different goalbox at low hunger. Next, all animals chose between these goalboxes in a Ushaped maze. Both choices were rewarded with food, and the animals were run under alternating conditions of high hunger and low hunger. Since the goalboxes were not visible from the choice point of the U-maze, a new, directional response was necessary to reach each goalbox. If high deprivation increases the strength of conditioning of a cue-reinforcer expectancy to stimuli that precede the reward, the rats should show an initial preference for the arm of the U-maze leading to the goalbox that had been previously paired with high-hunger food reward. Assuming that contrast is produced by the occurrence of reward magnitude different from that anticipated (Black, 1965; Bower, 1961), the preference for the high-hunger goalbox should subsequently be replaced by a preference for the low-hunger goalbox. On low-deprivation test sessions, the goalbox that previously had been paired with high-deprivation food reward would elicit a stronger cue-reinforcer expectancy than appropriate to the value of the current food reward. Therefore, the preference for this goalbox should be reduced. On high-deprivation test sessions, the other goalbox, which pre- viously had been paired with low-deprivation food reward, should elicit a lower level of cue-reinforcer expectancy than appropriate to the current reward value of the food. Therefore, the preference for this goalbox should be increased.

\section{Method}

Subjects and Apparatus. Twenty-four male hooded rats, approximately 100 days old and housed in groups of three, served as subjects. The training apparatus was a runway consisting of a $22 \times 12 \times 23 \mathrm{~cm}$ startbox, a $91 \times 10 \times 23 \mathrm{~cm}$ straight alley, and a $30 \times 12 \times 23 \mathrm{~cm}$ goalbox. The plywood floor and walls of the runway were painted flat gray. Wire mesh covered the floor and also served as a roof. Movable guillotine doors separated the startbox from the alley, and the alley from the goalbox. A gray cloth curtain was draped across the entrance to the goalbox, preventing sight of the contents of the goalbox prior to entry. A $5 \times$ $5 \times .6 \mathrm{~cm}$ wooden food cup was attached to the goalbox floor adjacent to the rear wall. Two goalbox liners, one black and the other striated vertically with black stripes and white stripes, could be inserted to cover the goalbox walls of either the training apparatus or the test apparatus. The test apparatus was a plywood U-maze, painted flat gray, consisting of a $22 \times 10 \times 23 \mathrm{~cm}$ startbox, a $25 \times 10 \times 23 \mathrm{~cm}$ stem, an $86 \times 10 \times 23 \mathrm{~cm}$ crossbar, two $91 \times 10 \times 23 \mathrm{~cm}$ goal alleys perpendicular to the crossbar, and two $30 \times 12 \times 23 \mathrm{~cm}$ goalboxes. Wire mesh served as the roof of the maze and covered the wooden floor. The subjects could not view the cloth curtains covering the goalboxes until after a choice response had been made. The contents of the goalboxes could not be viewed prior to entry. Guillotine doors separated the stem from the startbox, each side of the crossbar from the stem, and the goal alleys from the goalboxes. A $5 \times 5 \times .6 \mathrm{~cm}$ wooden food cup was attached to each goalbox floor adjacent to the rear wall.

Procedure. For all rats throughout the experiment, feeding periods of $22 \mathrm{~h}$ alternated with deprivation periods of $26 \mathrm{~h}$. At the start of each feeding period, a sufficient number of food pellets were placed on the floor of each home cage so that some pellets always remained for removal at the end of the feeding period. On each of the first 2 days of pretraining, the rats, in groups of three, were given 2 min of exploration of the runway with the guillotine doors and goalbox curtain open. On Days 3-10, each animal was placed in the goalbox for 2 min each day with the wall patterns absent and with four $37-\mathrm{mg}$ Noyes pellets present in the food cup. These placements occurred at the same time each day, the level of deprivation at placement alternating from day to day between 2-4 and 22-24 h. By the 9th day of placements, every animal was consuming the entire reward regardless of deprivation condition. Runway training to a four-pellet reward was begun on Day 11. As in pretraining, the rats were run alternatively, from day to day, 2-4 $\mathrm{h}$ food deprived and 22-24 $\mathrm{h}$ food deprived. For half the rats, high-deprivation runs ended in the black goalbox and low-deprivation runs ended in the striped goalbox, the reverse pairing being used for the remaining rats. Half the rats received their first day's runway trials at high deprivation; the remaining rats received theirs at low deprivation. Except where otherwise noted, the following procedure was used on each trial. Each animal was first placed in the startbox. Fifteen seconds later, the door to the alley was opened. Doors were closed behind the animal when it entered the alley and then the goalbox. Following $30 \mathrm{sec}$ in the goalbox, the animal was returned to the startbox, and then the preceding procedure was repeated. If an additional trial was not scheduled, the rat was carried directly from the goalbox to its home cage. Since the number of failures to completely consume the reward was negligible during training and test, the experimental results to be reported cannot be attributed to a pairing of the two goalbox patterns with different quantities of consumed food. On the first trial of the first four sessions only, each animal was placed directly into the goalbox for $30 \mathrm{sec}$. For these direct placements, the goalbox contained the appropriate wall pattern 
and the four-pellet reward. On the remaining two trials of each of the first two sessions and the remaining five trials of the next two sessions, the regular procedure was adhered to. The rats next received five trials on each of 14 daily sessions, making a total of 88 training trials.

Preference testing in the U-maze followed the completion of training. The black liner was inserted into the left goalbox, and the striped liner was inserted into the right goalbox. It will be recalled that for half the subjects the black liner had been paired in training with the receipt of reward at high hunger, the reverse pairing occurring for the remaining subjects. Each rat received six test trials per day on 10 consecutive days. The closing of the appropriate choice-point door forced the animals to run once to each goalbox on the first two trials of each daily session. This was followed by four free-choice trials. The direction of the first forced trial varied across days according to the repeated pattern: RLLRRL. Every goalbox entry was rewarded with four $37-\mathrm{mg}$ pellets. On all trials, guillotine doors on either side of the choice point and at goalbox entrances were lowered behind the animals to prevent retracing. As in training, the rats were run alternately from day to day 2-4 and 22-24 h food deprived. Other details of testing were the same as those used in training.

\section{Results and Discussion}

During the course of training, one rat for whom the black goalbox was paired with high-hunger reward was eliminated for failing to traverse the runway within 5 min on any trial for 5 consecutive days. A second rat, for whom the striped goalbox was paired with high-hunger reward, was eliminated during training for extreme emotionality.

The predicted contrast effect occurred. Early in the test, the black goalbox was chosen more frequently by the group that had been trained with highdeprivation reward in the black goalbox than by the group that had received the low-hunger reward in the black goalbox, followed by the reversal of this preference later in the test (see Figure 2). A $2 \times 5$ (group $x$ blocks of eight trials) repeated-measures analysis of variance revealed significant effects for groups $[F(1,20)=8.66, p<.005]$ and the groups $\times$ trial block interaction $[F(4,80)=12.11, \mathrm{p}<.001]$. A simple main effects analysis was used to break down the interaction. The reported significance levels for the simple main effects are conservative since they were adjusted as recommended by Kirk (1968) for multiple comparisons. On the first block of test trials, the group which in training had had the black goalbox paired with high-hunger reward chose the black goalbox significantly more often than did the group which in training had had the striped goalbox paired with high-hunger reward $[F(1,100)=7.31, p<.05]$. This difference was no longer present on the second block of trials $[F(1,100)=1.14]$. On each subsequent trial block, the rats for which the black goalbox had previously been paired with high-deprivation reward preferred the black goalbox less than did the rats that had received the opposite pairing $[\mathrm{Fs}(1,100)=14.73$, $33.13,24.04$, respectively, ps $<.001]$.

The preceding statistical design, which controls for

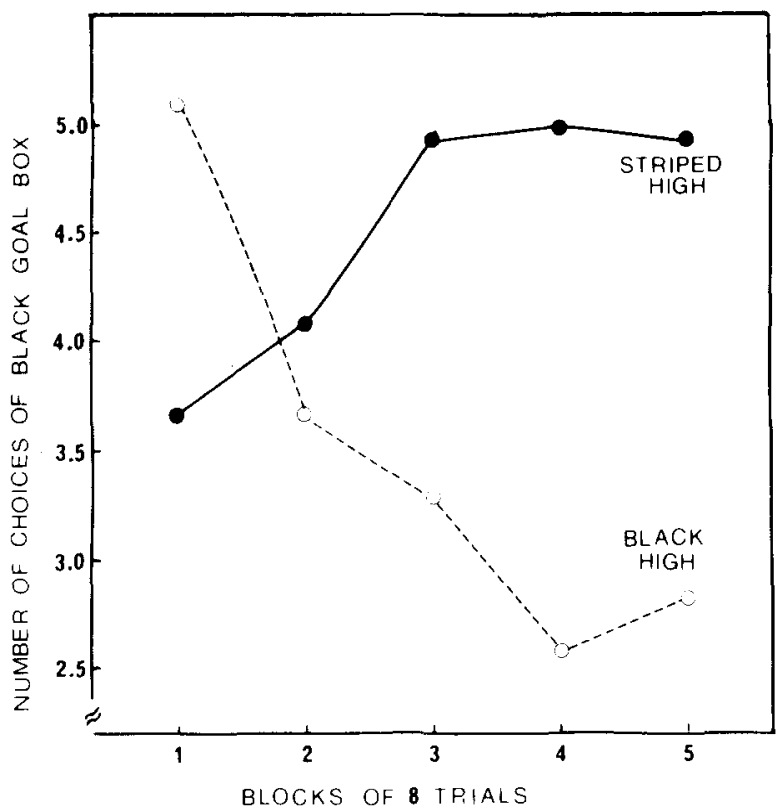

Figure 2. Mean number of choices, in blocks of eight trials, of the black goalbox by the group which in training had the black goalbox paired with high-hunger food reward (black high) and the group which in training had the striped goalbox paired with highhunger food reward (striped high).

a preexperimental preference of the rat population for one goalbox over the other (e.g., Eisenberger, Myers, Sanders, \& Shanab, 1970), will be discussed in some detail as an aid to researchers working with choice behavior and to readers who may wonder why we avoided the more straightforward statistical design of comparing the average preference for the high-deprivation goalbox against the chance level of $50 \%$. After all, one might argue, any general preexperimental preference in the population for one of the two goalboxes was eliminated as a procedural confound by pairing the black goalbox with highhunger reward for half the rats and pairing the striped goalbox with high-hunger reward for the remaining rats. Note, however, that procedural counterbalancing does not eliminate the counterbalanced variable as a source of error variance in the statistical analysis. For example, let us say that the rat population had a pretreatment preference for the black goalbox rather than the striped goalbox. Assume that three hypothetical rats in the present experiment had black paired in training with high-hunger reward and that, in the test, the total numbers of choices of black by the rats were 8,9 , and 10 of a possible total of, say, 10. Say that three other rats had striped paired in training with high-hunger reward, and these rats chose black 6,7 , and 8 times. For the total sample of six rats, the mean number of choices of the highhunger goalbox would be six, as compared with the chance level of five. To determine whether there was 
a significant preference for the high-hunger goalbox, we can lump the six rats into a single group for which the mean performance can be compared with chance, using a one-group $t$ test. The result is a standard deviation of 3.4 , with $\mathrm{t}(5)=.719, \mathrm{p}<.7$. On the other hand, using precisely the same data, we can statistically remove an error variance due to the rat population's preexperimental preference for one goalbox over the other. This can be accomplished by rephrasing the experimental hypothesis to ask whether the preference for one of the two goalboxes (e.g., black) differs for two groups defined by the treatment (black vs. white paired with high-hunger reward in training). Retaining the six scores in our hypothetical sample, one obtains a difference between the means of 2 , a standard deviation of 1 , and $t(4)=2.45$, $\mathrm{p}<.10$. This example shows that the power of the second phasing of the hypothesis is greater than that of the first, an occurrence that results from the removal of error variance associated with the subject population's preexperimental preference for one choice over the other.

For those readers who, despite the preceding argument, prefer the one-group statistical design, with the number of choices of the high-hunger goalbox as the dependent measure: the high-hunger goalbox was selected a significantly greater number of times than chance on the first block of test trials and less than chance on the last block of test trials $[\operatorname{ts}(21)=3.04$ and 3.35 , respectively, ps $<.01]$.

\section{GENERAL DISCUSSION}

Deprivation shifts, using conditions analogous to those which produce strong incentive contrast when reward quantity is changed (extended preshift training, short interval from the last preshift trial to the first postshift trial, large preshift differences in deprivation), resulted in contrast. In the first experiment, a downshift of hunger produced a slow lessening of runway speed to below that of the constantlow-deprivation group. Although the occurrence of contrast was consistent with the view that deprivation affects the incentive value of the reinforcer, the effect developed more slowly than does that usually produced by shifts of reward quantity.

To explain this slow development of contrast, we suggest that deprivation level affects both a cuereinforcer expectancy and either habit strength or response-reinforcer expectancy. According to this view, high hunger produces a greater reward value of food and a greater cue-reinforcer expectancy and habit strength (or response-reinforcer expectancy) than does low hunger. Following training sufficient to establish a strong cue-reinforcer expectancy, a deprivation downshift would produce only a slow development of contrast, since the decremental effects of the less-than-expected reward value of food would initially be countered by generalized habit strength or response-reinforcer expectancy. As habit strength or response-reinforcer expectancy lessened to the value appropriate to the new deprivation level, contrast would occur.

The second experiment extended to deprivation shifts: (1) Spear and Spitzner's (1966) finding that pairing a change of reward quantity with a new instrumental requirement produces contrast, and (2) contrast of choice behavior (Eisenberger et al., 1975). In order to separate possible effects of deprivation upon the cue-reinforcer expectancy vs. habit strength or response-reinforcer expectancy, rats in the second experiment were given a choice between a goalbox that previously had been paired with food at high hunger vs. another goalbox which had been paired with food at low hunger. Since the goalboxes were not visible from the choice point, preferences reflect the reward value of the goalbox stimuli rather than processes related to the previously learned approach responses to the goalboxes. There was an initial preference for the high-hunger goalbox, suggesting that the level of hunger at which an exteroceptive stimulus is paired with food contributes to its secondary reward value. Preference then changed to the lowhunger goalbox, indicating contrast of choice behavior.

The present results suggest that a cue-reinforcer expectancy together with either habit formation or response-reinforcer expectancy may have contributed to the residual deprivation effects found by Kurtz and Jarka (1968) and Revusky (1967, 1978). Kurtz and Jarka gave rats 16 choice trials in a T-maze. On high-hunger trials, one choice produced food, while on low-hunger trials, the alternative choice yielded food. Interspersed with the free-choice trials were 60 rewarded forced trials, half to each goal arm at the appropriate hunger level. Seventy percent of all choices at either hunger level were of the goal arm to which the animals had been forced at the greater hunger. Revusky (1968, Experiment 2) had rats consume a small quantity of flavored water $2 \mathrm{~h}$ prior to delivery of the daily maintenance supply of water, and an equal quantity of a different flavor following the maintenance watering. The flavor presented at high thirst to half the rats was presented at low thirst to the remaining rats. All rats were then given free access to both solutions at either thirst level. At both levels of test thirst, the relative amount consumed of a flavor was greater if it had originally been presented at the higher thirst level. A similar effect was found for hunger (Revusky, 1967). According to the present view, the greater in-test consumption of the food or drink with the high-deprivation flavor was the result both of a stronger drink habit or response-reinforcer expectancy and a greater reward expectancy. 


\section{REFERENCES}

AMsel, A. The role of frustrative nonreward in noncontinuous reward situations. Psychological Bulletin, 1958, 55, 102-119.

Br.ACK, R. W. On the combination of drive and incentive motivation. Psychological Review, 1965, 72, 310-317.

Bolles, R. C. Comments on Professor Spence's paper. In M. R. Jones (Ed.), Nebraska Symposium on Motivation. Lincoln: University of Nebraska Press, 1958.

Bolles, R. C. Reinforcement, expectancy, and learning. Psychological Review, 1972, 79, 394-409.

Boltes, R. C. Theory of motivation (2nd ed.). New York: Harper \& Row, 1975.

Bower, G. H. A contrast effect in differential conditioning. Journal of Experimental Psychology, 1961, 62, 196-199.

Brush, F. R., Goodrich, K. P., Teghtsoonian, R., \& Eisman, E. H. Dependence of learning (habit) in the runway under three levels of sucrose incentive. Psychological Reports, 1963, 12, 375-384.

Butter, C. M., \& Campbell, B. A. Running speed as a function of successive reversals in hunger drive level. Journal of Comparative and Physiological Psychology, 1960, 53, 52-54.

CAPAldi, E. D. Simultaneous shifts in reward magnitude and level of food deprivation. Psychonomic Science, 1971, 23, 357359.

CAPAldi, E. D. Resistance to extinction in rats as a function of deprivation level and schedule of reward in acquisition. Journal of Comparative and Physiological Psychology, 1972, 79, 90-98.

Capaldi, E. D. Effects of shifts in body weight on rats' straight alley performance as a function of reward magnitude. Learning and Motivation, 1973, 4, 229-235.

Capaldi, E. D., \& HovanciK, J. R. Effects of previous body weight level on rats' straight-alley performance. Journal of Experimental Psychology, 1973, 97, 93-97.

Capaldi, E. D., Smith, N. S., \& White, L. A. Control of reward expectancies by drive stimuli. Journal of Experimental Psychology: Animal Behavior Processes, 1978, 3, 178-188.

CoHen, J. M. Drive level effects on the conditioning of frustration. Journal of Experimental Psychology, 1973, 98, 297-301.

Deese, J., \& Carpenter, J. A. Drive level and reinforcement. Journal of Experimental Psychology, 1951, 42, 236-238.

Di Lollo, V., \& Beez, V. Negative contrast effect as a function of reward decrement. Psychonomic Science, 1966, 5, 99-100.

Eisenberger, R., Frank, M., \& Park, D. C. Incentive contrast of choice behavior. Journal of Experimental Psychology: Animal Behavior Processes, 1975, 4, 346-354.

Eisenberger, R., Myers, A. K., \& Kaplan, R. M. Persistent deprivation-shift effect opposite in direction to incentive contrast. Journal of Experimental Psychology, 1973, 99, 400-404.
Eisenberger, R., Myers, A. K., Sanders, R., \& Shanab, M. Stimulus control of spontaneous alternation in the rat. Journal of Comparative and Physiological Psychology, 1970, 70, 136-140.

Gleitman, H., \& Steinman, F. Depression effect as a function of the retention interval before and after shift in reward magnitude. Journal of Comparative and Physiological Psychology, $1964,57,158-160$.

Hovancik, J. R. The effect of deprivation level during noncontingent pairings and instrumental learning on subsequent instrumental performance. Learning and Motivation, 1978, 9, $1-15$.

KinK, R. E. Experimental design. Belmont, Calif: Wadsworth, 1968.

Kuntz, K., \& JARKA, R. G. Position preference based on differential food privation. Journal of Comparative and Physiological Psychology, 1968, 66, 518-521.

Mollenauer, S. O. Shifts in deprivation level: Different effects depending on amount of pre-shift training. Learning and Motivation, 1971, 2, 58-66.

Moltz, H., \& MAdDi, S. R. Reduction of secondary reward value as a function of drive strength during latent extinction. Journal of Experimental Psychology, 1956, 52, 71-76.

Pliskofr, S. S. Response elimination as a function of generalization, motivation and number of non-response extinction trials. Unpublished doctoral dissertation, New York University, 1955.

REvusky, S. H. Hunger level during food consumption: Effects on subsequent preference. Psychonomic Science, 1967, 7, 109110.

Revusky, S. H. Effects of thirst level during consumption of flavored water on subsequent preference. Journal of Comparative and Physiologica! Psychology, 1968, 66, 777-779.

Spear, N. E., \& Spitzner, J. H. Simultaneous and successive contrast effects of reward magnitude in selective learning. Psychological Monographs, 1966, 80(10, Whole No. 618).

Spence, K. W. Behavior theory and conditioning. New Haven: Yale University Press, 1956.

TimBerlake, W. Straight alley acquisition drive and ad lib test performance. Psychonomic Science, 1967, 9, 585-586.

Vogel, J. R., Mikulka, P. J., \& Spear, N. E. Effect of interpolated extinction and level of training on the "depression effect." Journal of Experimental Psychology, 1966, 72, 51-60.

ZARETSKY, H. H. Learning and performance in the runway as a function of the shift drive and incentive. Journal of Comparative and Physiological Psychology, 1966, 62, 218-221.

(Manuscript received April 7, 1981; revision accepted for publication July 21, 1981.) 